\title{
Sensitivity of geomagnetically induced currents to varying auroral electrojet and conductivity models
}

\author{
Ciarán D Beggan
}

\begin{abstract}
Geomagnetically induced currents $(\mathrm{GIC})$ are created by the interaction of rapid changes in the magnitude of the magnetic field with the conductive subsurface of the Earth. The changing magnetic field induces electric currents, which are particularly strong along boundaries between regions of contrasting conductivity structure such as the land and sea. A technique known as the 'thin-sheet approximation' can be used to determine the electric field at the Earth's surface, which in turn allows the calculation of GIC in the earthing connections of high-voltage nodes within a power grid. The thin-sheet approximation uses a spatially varying conductance over the region of interest on a 2D surface, combined with a 1D layered model of upper lithosphere conductance. We produce synthetic models of the auroral electrojet in different locations over the United Kingdom (UK) and investigate the effects of varying the 2D thin-sheet model. We assess different two-dimensional surface conductance models and vary the underlying 1D conductivity models to simulate the effects of resistant through to conductive lithosphere. With an advanced network model of high-voltage electrical distribution grid, we compute the expected GIC at each node in the system given the input surface electric fields from the various synthetic electrojets and conductivity models. We find that the electrojet location is the primary control on the size of GIC, with conductivity being a second-order effect in general, though it can be locally important.
\end{abstract}

Keywords: Geomagnetically induced currents; Ground conductivity; Electric field

\section{Background}

Electric fields are generated in the ground during large space weather events primarily due to the induction effects of a changing magnetic field within a conductive medium (i.e. the subsurface). During geomagnetic variations, geomagnetically induced currents (GIC) can flow in certain types of grounded systems such as long pipelines and electrical distribution systems. These currents are usually harmless but high-voltage power systems can be vulnerable to GIC flow if they offer a low-resistance path for the currents compared to the ground itself (Boteler et al. 2013; Pirjola 2005; Viljanen and Pirjola 1994). The social and economic impacts of an extreme space weather event could potentially be very large if were to cause damage to power transmission networks (c.f. Radasky 2011; Schulte in den Bäumen et al. 2014), and hence, large GIC

Correspondence: ciar@bgs.ac.uk

British Geological Survey, West Mains Road, EH9 3LA Edinburgh, UK are of concern to network operators and civil government, amongst others (Cannon 2013; Thomson 2014).

Studies of past space weather events in the United Kingdom (UK), such as McKay (2003) and Turnbull (2010, 2011), have modelled the impacts on simplified versions of the high-voltage transmission system in the UK, using basic representations of the conductivity structure of the land and bathymetry of the offshore continental shelf (Beamish et al. 2002). These models were able to reproduce GIC values measured in Scotland during the October 2003 storm (Thomson et al. 2005). Measured GIC (three phases summed) in the UK during the October 2003 event reached a peak of $40 \mathrm{~A}$ in one high-voltage node.

More recently, Pulkkinen et al. (2012) developed scenarios of realistic electric field changes for a 100-year extreme event to aid network engineers and planners. These were applied to the high-voltage network of Virginia in the USA (see also Bernabeu 2013) and to a relatively simple model of the UK high-voltage network to compute

\section{Springer}


the expected GIC. In these models, GIC of up to $300 \mathrm{~A}$ were found. A one-dimensional conductivity depth model was used giving a uniform electric field model; however, it is expected, in reality, that large-scale spatial variations would occur during a significant geomagnetic storm. Viljanen et al. (2014) have also produced a model of the European high-voltage network as part of the EURISGIC project (Viljanen 2011) from which they computed GIC of up to $100 \mathrm{~A}$, again using a simple conductivity structure for the British Isles.

In order to increase the complexity of the geophysical and engineering layers, Beggan et al. (2013) investigated the use of a more sophisticated high-voltage electrical network model coupled to a more representative two dimensional surface conductance model, as developed by Beamish and White (2012). These improvements were applied to a set of reasonable 'worst-case' magnetic field change scenarios for the UK mainland, based on work from Thomson et al. (2011). Values of up to 460 A were modelled in one node for an extreme 200-year return event.

These large GIC values were generated with a fixed set of conductivity model parameters. Until now, there has been little investigation into the sensitivity of the GIC values to variation in the geophysical conductance models used to create them. Previously, extreme endmember states were chosen, and intermediary values were assumed to vary linearly. The aim of this study is to check if these assumptions are valid for the sophisticated models that are currently being employed. To do so, we examine the differences between GIC values that arise when different 2D surface conductance models are used and when the underlying $1 \mathrm{D}$ depth model is varied. In the next section, we outline the models used and the methodology for computing GIC.

\section{Methods}

\section{Computing models of GIC}

To compute GIC within an electrical network, three main components are required: (1) a model of the conductivity structure of the region; (2) spatial and temporal measurements of the magnetic field; and (3) information about the topology and electrical properties of the high-voltage network. As we are primarily interested in how the conductivity affects GIC, we focus initially on the details of the one-dimensional depth and two-dimensional surface conductance models, before describing the synthetic auroral electrojets and high-voltage network model.

\section{Conductivity models}

The diffusion length of the magnetic field into the subsurface (skin depth) is dependent on the conductivity of the local region and the time period (frequency) over which the change of the magnetic field occurs. The vertical distribution of the resistivity within the Earth's crust, and the period considered, determine the size of the induced electric field. Deeper layers are more significant at long periods, while shallow layers have a stronger influence at short periods. The interaction of the external magnetic field with the conductive Earth can be approximated by thin-sheet modelling and the frequency (i.e. period) of the rate of change of the magnetic field, which relates to the penetration depth.

The thin-sheet modelling code used in this study is based upon the work of Vasseur and Weidelt (1977) and has been used in several previous studies (c.f. McKay 2003; Thomson et al. 2005). The code determines the surface electric field arising at a particular frequency from conductivity models of the surface and subsurface. Using a series of Green's functions and integrals, a two-dimensional thin-sheet approximation can be used to model the effect that conductivity variations have on redistributing regional or 'normal' currents induced elsewhere (for example, in the shallow sea). The surface layer can be regarded as an infinitely thin sheet of finite laterally variable conductance, across which certain boundary conditions apply. A horizontal magnetic field will induce an electric field in the subsurface which creates a discontinuity current sheet at the surface. Hence, the thin-sheet model includes the effect that lateral conductivity variations will have on redistributing regional currents induced elsewhere. In this study, we analyse three different thinsheet models, shown in Figure 1. All have a grid cell resolution of $10 \mathrm{~km}$, covering an area of $1,200 \times 1,700 \mathrm{~km}$. In practice, we assume the $2 \mathrm{D}$ conductance represents the depth-integrated conductivity of the upper $3 \mathrm{~km}$ of the crust.

The first 2D model (termed the 'Constant' model) is a simple land-sea model (Figure 1A). The depth-integrated conductivity (i.e. conductance) of the land is set to $20 \mathrm{~S}$, while the conductance of sea water is set to $600 \mathrm{~S}$. The second model is based on the work of McKay (2003) and is called the 'AMK2003' model (Figure 1B). This model has a more sophisticated bathymetric representation around the shallow continental shelf using a uniform value of sea water conductivity $(4 \mathrm{~S} / \mathrm{m})$. Most of the land area is set to $20 \mathrm{~S}$, but conductivity information from long-period magnetotelluric studies of the region around southern Scotland and northern England is included in these areas (e.g. Banks et al. 1983).

In 2012, a new UK thin-sheet conductivity model (termed 'BGS2012') was derived from the analysis of the resistivity properties of bedrock materials based on the British Geological Survey (BGS) 1:625,000 geological map of the UK and Northern Ireland (Figure 1C). This model uses information obtained from airborne geophysical surveys across the UK to provide a lithological and geostatistical estimate for all the UK bedrock formations. 

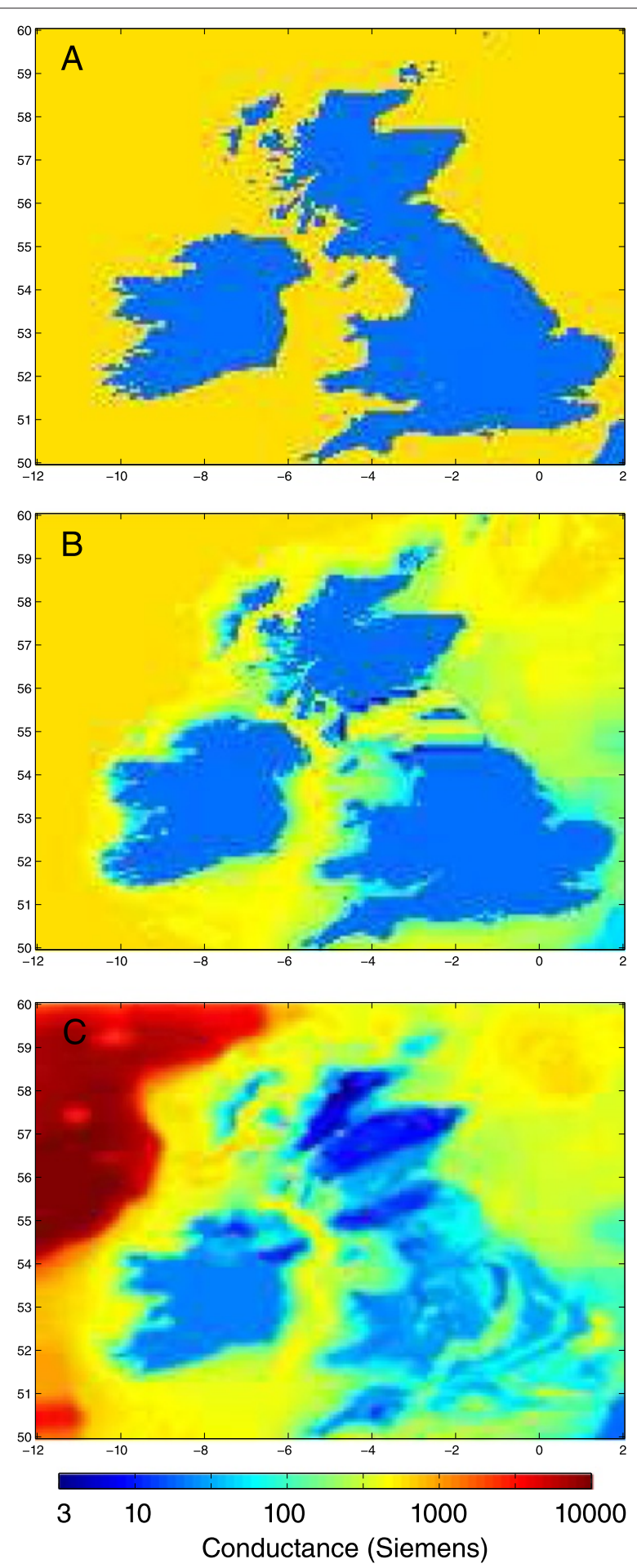

Figure 1 Two-dimensional conductance models. (A) Constant model, (B) AMK2003 model and (C) BGS2012 model. See text for details.

The central moments of the distributions were found to range from 8 to $3,125 \Omega \mathrm{m}$ (Beamish 2012). Over the continental shelf and ocean, a more complex bathymetry map was used, leading to a maximum conductance of approximately $1,600 \mathrm{~S}$ for the deeper parts of the Atlantic ocean. Onshore, the conductance values are for a depth integration of $3 \mathrm{~km}$. The map differs strongly from the AMK2003 in the region around southern Scotland and northern England, as it relates to the shallow subsurface response as inferred from aerial survey but is selfconsistent across the UK.

In addition to the two-dimensional surface conductivity, the thin-sheet modelling code also requires a layered one-dimensional conductivity model at depth. We construct six representative models for the conductance of the upper crust across the UK and a seventh representing a homogeneous half-space. The models are based on measurements made by various groups in the UK from 1977 to 1996 (see Banks et al. 1996; McKay 2003, and references therein), though applied across the UK as a whole for the purposes of this study.

Table 1 shows the values for the upper $30 \mathrm{~km}$ of each model. The upper $30 \mathrm{~km}$ vary between the six example models from 20 to $10,000 \Omega \mathrm{m}$. The lower layers of the models have the same fixed values (Table 2) down to a depth of $390 \mathrm{~km}$. A homogeneous half-space model with a constant value of $900 \Omega \mathrm{m}$ with no depth variation is also used; this has an average similar to the General Model. Deeper conductivity information may be added, if desired, but given the relatively short periods of magnetic field change, we investigate (see next section) and we do not need deeper layers, as depth penetration is less than a few hundred kilometers.

In terms of geological settings, the Northern Highlands model represents a very resistive terrane, found in northern Scotland, where the underlying lithology consists of granitic or metamorphic rocks. The Central Highlands are a less resistive version of this model. The Southern Uplands has a very conductive upper lithosphere, while the General Model is an intermediary between these endmember regions. The other models types examine the effect of a hidden resistive layer (Concealed Caledonides) or a more typical sedimentary basin setting (Midland Valley), common across the central and southern UK. The

Table 1 One-dimensional resistivity models: 0 to $30 \mathrm{~km}$ [units: $\Omega \mathbf{~ m}$ ]

\begin{tabular}{lrrr}
\hline Depth (km) & $\mathbf{0}$ to $\mathbf{1 0}$ & $\mathbf{1 0}$ to $\mathbf{2 0}$ & $\mathbf{2 0}$ to $\mathbf{3 0}$ \\
\hline Northern Highlands (NH) & 10,000 & 5,000 & 2,000 \\
Central Highlands (CH) & 1,000 & 1,000 & 200 \\
Midland Valley (MV) & 100 & 150 & 250 \\
Southern Uplands (SU) & 20 & 20 & 1,000 \\
Concealed Caledonides (CC) & 500 & 2,000 & 500 \\
General Model (GM) & 1,000 & 1,000 & 500 \\
Homogeneous half-space (HH) & 900 & 900 & 900 \\
\hline
\end{tabular}


Table 2 One-dimensional resistivity models: 30 to $390 \mathrm{~km}$ [units: $\Omega \mathrm{m}$ ]

\begin{tabular}{lrr}
\hline Depth $(\mathbf{k m})$ & All models & Homog. Halfsp. \\
\hline 30 to 40 & 112.4 & 900 \\
40 to 50 & 100.0 & 900 \\
50 to 60 & 89.3 & 900 \\
60 to 70 & 82.3 & 900 \\
70 to 80 & 79.4 & 900 \\
80 to 90 & 70.9 & 900 \\
90 to 190 & 63.3 & 900 \\
190 to 290 & 50.0 & 900 \\
290 to 390 & 31.3 & 900 \\
\hline
\end{tabular}

homogeneous half-space is a simple 1D moderately resistive model. Note that combining the three 2D surface models combined with the seven $1 \mathrm{D}$ depth models gives 21 different conductivity scenarios to assess.

\section{Magnetic field models}

To simulate the magnetic field during a geomagnetic storm, we created a series of representations of the magnetic field arising from a synthetic electrojet current system moving across the UK as the auroral oval expands southward. We constructed four simulated 'electrojet' models, all approximately $800 \mathrm{~km}$ wide, with a tapered-cosine form. Three of the models have an orientation approximately geomagnetically east-west aligned across the UK, placed at three different latitudes. The first lies over northern UK ('northern Scotland'), the second over the central UK ('northern England') and the third along the southern coast (passing through 'London'). For completeness, an orthogonal magnetic field direction in a geomagnetic north-south alignment (approximately following the central axis of the 'Irish Sea') was also created. Although such an orientation is not realistic due to the configuration of the main magnetic field, storms can exhibit a locally strong north-south component for short periods. The electrojet models were created as normalized values on a grid in geomagnetic coordinates and then rotated $10^{\circ}$ counterclockwise to match the appropriate position over the UK in geographical coordinates. The electrojet grids were cropped and subsampled to 1/12th of a degree $(\approx 10 \mathrm{~km})$ to match the grid spacing of the $2 \mathrm{D}$ thin-sheet conductivity models.

In order to compute the electric field response, the thin-sheet approximation code requires the average rate of change of the horizontal field over a fixed period. If we assume the amplitude of the horizontal field changes sinusoidally with a period of length $T$ (in minutes), as the electrojet moves back and forwards, the input field strength $\left(H_{0}\right)$ at any time $(t)$ can be represented by:

$$
\mathbf{B}_{H}=H_{0} \sin (2 \pi t / T)
$$

We wish to compute the average rate of change of this equation. If we differentiate and then assume that the root-mean-square value of a sinusoid is $1 / \sqrt{2}$, then the horizontal rate of change can be written as:

$$
d \mathbf{B}_{H} / d t=H_{0} \frac{2 \pi}{T} \frac{1}{\sqrt{2}}=H_{0} \frac{\sqrt{2} \pi}{T}
$$

We mimic the expected 30-, 100- and 200-year extreme scenarios of the magnetic field (c.f. Beggan et al. 2013), by scaling the electrojet $H_{0}$ to 250,320 and $450 \mathrm{nT}$ for a period $(T)$ of $2 \mathrm{~min}$ and 1,250, 1,575 and 2,275 $\mathrm{nT}$ for a period $(T)$ of $10 \mathrm{~min}$, giving six different magnetic field strength models. The values are chosen from the work of Thomson et al. (2011) based on records from European observatories over the past 30 years. Note that these are only meant to be snapshots of the extreme rate of change - such values are generally not sustained for very long (e.g. tens of seconds).

As there are four electrojet positions with six variations of field strength, this gives 24 different electrojet models. Together with the 21 different combinations of conductivity models, this gives a total of 504 different electric field models that can be computed.

\section{Computing GIC}

Once the spatially varying surface electric field has been found from the thin-sheet calculations, GIC can be computed using information about the network topology and characteristics. This consists of the latitude and longitude positions, connections and the electrical characteristics (earthing, transformer and line resistance) of each node in the high-voltage network. These parameters are used to calculate GIC (I, in ampere) along power transmission lines from the standard matrix equation in Lehtinen and Pirjola (1985):

$$
\mathbf{I}=(\mathbf{1}+\mathbf{Y Z})^{-1} \cdot \mathbf{J}
$$

where $\mathbf{Z}$ is the impedance matrix, $\mathbf{Y}$ is the network admittance matrix and $\mathbf{1}$ is the identity matrix. $\mathbf{J}$ is the 'perfect earthing' current (in the case that $\mathbf{Z}=0$ ) and is defined as:

$$
J_{i}=\Sigma_{j \neq i} V_{i j} / R_{i j}
$$

The values of $V_{i j}$ are the geo-voltages between nodes $i$ and $j$ computed from the electric field map with $R_{i j}$ being the line resistance between the two nodes, taken from the network model. The network parameters are also used to calculate $\mathbf{Y}$ and $\mathbf{Z}$, and the geo-voltage is calculated by interpolating the electric field model values onto the positions of the transmission lines and integrating along a straight line between nodes. The GIC at each node on the grid is then computed using both the North and East components of the surface electric field. The vector I contains the estimated GIC for each node. 
As we wish to examine the sensitivity of the computed GIC to changing the conductivity models, we fix the properties of the network. This is a modified version of the 2012 model of the UK network (Beggan et al. 2013), consisting of 695 nodes and 1,178 connections. Figure 2 shows an overview of the National Grid UK high-voltage 400and 275-kV line distribution power network model. Additionally, in Scotland, National Grid operate the 132-kV network. Note also that multiple nodes (typically containing multiple transformers) can be co-located on the same site. The locations marked by triangles labelled A to L are the 12 nodes with the largest modelled GIC.

\section{Results}

Once the various electrojet and conductivity models were defined, we ran the thin-sheet modelling code for all 504 combinations of electrojet and conductivity model scenarios.

\section{Visual assessment of GIC}

Figure 3 gives some examples of the electric field models that have been generated. It is intended to show the variation that can occur from varying the one-dimensional and two-dimensional models, as well as the electrojet position. The top row shows examples of the four electrojet positions, scaled to 2,275 nT, i.e. a 200 -year extreme $H_{0}$ for a

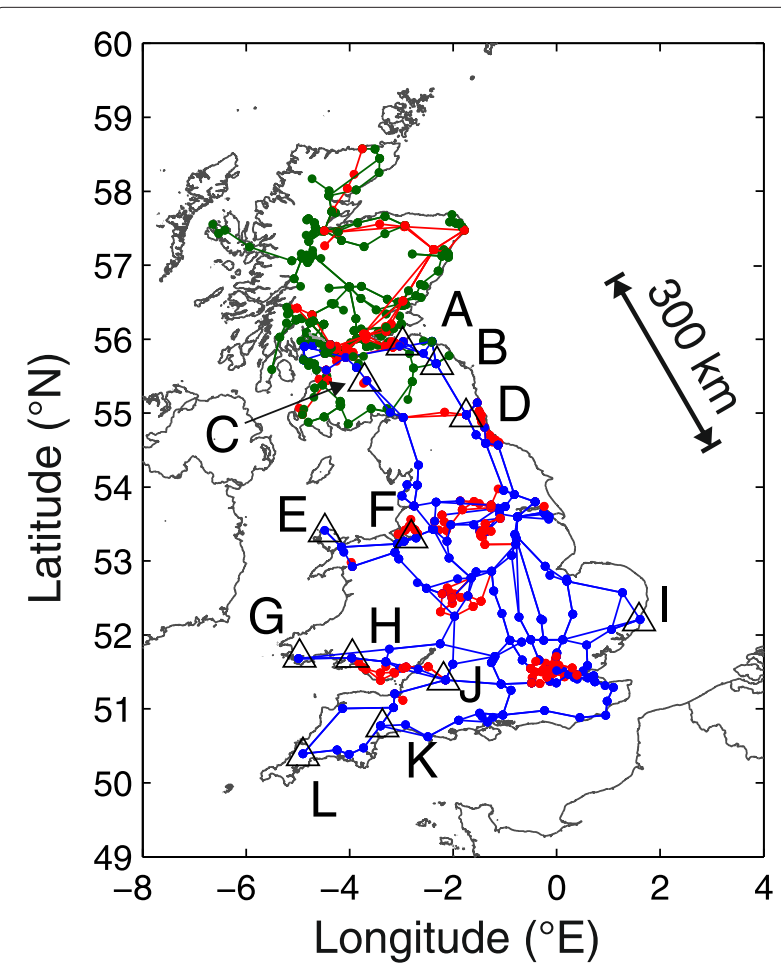

Figure 2 High-voltage network model for the UK mainland. Blue: 400 kV; Red: 275 kV; Green: 132 kV (Scotland only). Also shown are the locations of the top 12 nodes with largest GIC, labelled A to L. period of $10 \mathrm{~min}$. The electric fields from 12 selected conductivity model interactions (generated using the above electrojets) are shown in the lower three rows of Figure 3. The second row shows the electric fields using the Constant model, the third row is computed with the AMK2003 model, while the bottom row illustrates the output from the BGS2012 model. The columns are labelled with the 1D conductivity depth model used in combination with the 2D surface model. The first column (labelled $\mathrm{NH}$ ) shows the resistive Northern Highlands, the second (SU) shows the conductive Southern Uplands, the third column (GM) is the output from General Model, while the rightmost $(\mathrm{HH})$ is the homogeneous half-space. As can be observed in Figure 3, the largest electric field values $(>7 \mathrm{~V} / \mathrm{km})$ are generated when the electrojet is orientated approximately north-south, with the homogeneous half-space. The Southern Uplands model generates the smallest electric field values.

From the 504 electric field models, GIC at each of the 695 nodes was computed. Figure 4 shows examples of GIC calculated from some of these electric field models. A single $1 \mathrm{D}$ conductivity model (the General Model) was used. GIC for the Constant, AMK2003 and BGS2012 2D models using three electrojet locations are shown. The first column is the GIC computed for each conductivity model when the electrojet is located over Northern Scotland. Large GIC do appear in the central and southern parts of the UK and particularly along the coast. The size of the GIC are slightly reduced in the central regions of Scotland in the AMK2003 model, as the conductivity here is different compared to the Constant and BGS2012 model.

With the electrojet located over the London region, GIC are reduced compared to the Northern Scotland electrojet location models, though are still large around the central UK on the eastern coast. From examining the differences between the models in this figure, it suggests that GIC primarily responds to the location of the electrojet, though there is some local variation due to regional conductivity differences (e.g. in the central region of Scotland in the AMK2003 model).

Figure 2 shows the locations of the 12 nodes with the largest average GIC for these particular scenarios and network topology. The nodes with the largest GIC are typically at the end of long lines or 'corner' nodes in the network but note that different nodes can become larger depending on the network model used. All chosen nodes record GIC values of over $80 \mathrm{~A}$, with one over $225 \mathrm{~A}$ for the General Model with the electrojet across North Scotland (node B).

From Figure 4, it is clear that when the electrojet model is over London, there are still relatively large GIC in the central Scotland area. Equally, when the electrojet is located over northern Scotland, there are some nodes with significant GIC (> $25 \mathrm{~A}$ ) in the southerly parts of the UK. 


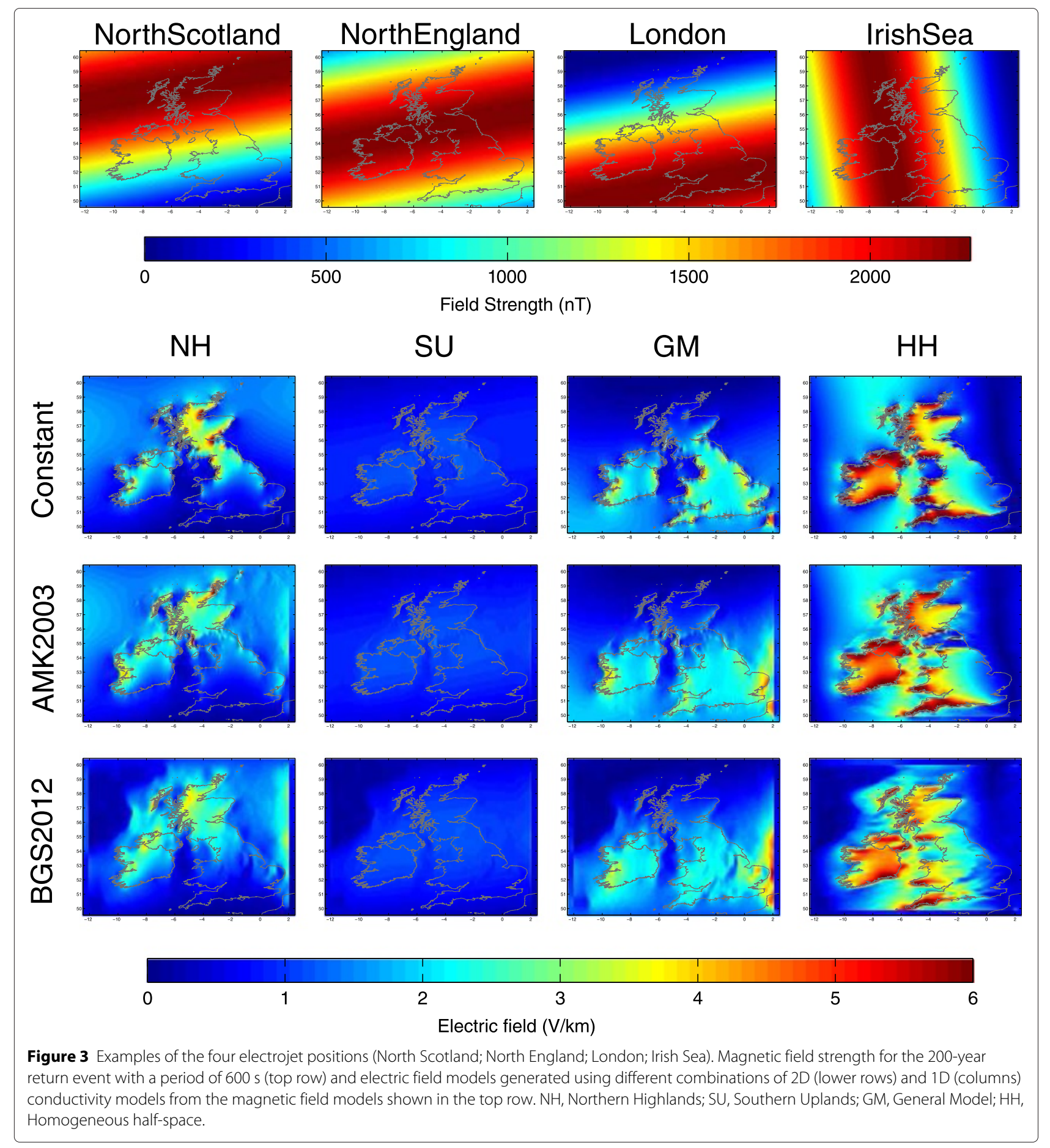

This suggests that the impact of the electrojet can be dispersed from the source region to elsewhere in the country via the high-voltage network.

The UK network is essentially composed of seven regions, as a consequence of the historic evolution of the grid. There are dense concentrations of nodes around population centres, with a series of long lines running between clusters of generators in central England and central Scotland. This allows currents to equalise over long distances from regions of high electric field to lower electric field over the high-voltage network. Thus, nodes in locations of low electric field may experience relatively large GIC, as they offer an easier route to normalization of current. 

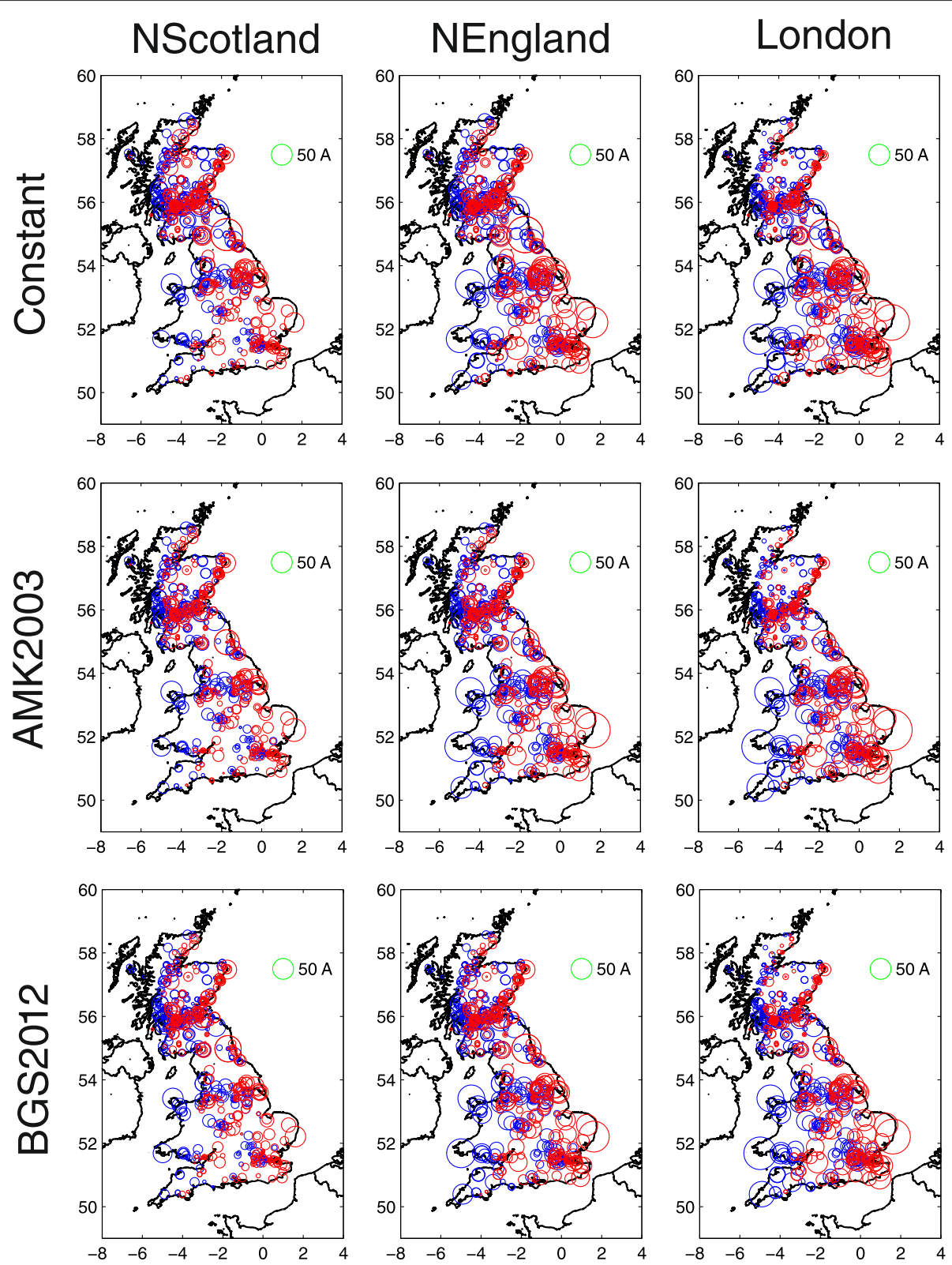

Figure 4 Example GIC generated from three of electrojet positions (North Scotland; Northern England; London). GIC for the 200-year return event with a period of $600 \mathrm{~s}$ and electric field models generated using different combinations of 2D (rows) conductivity models and the 1D General Model. Circle size indicates GIC relative to the 50-A scale. Blue colour shows nodes where current enters the grid; red shows current entering the ground.

\section{Analysis of variance}

Attempting to visually analyse the GIC from all 504 electric field models is rather difficult. Indeed, we wish to understand the relative influence and importance of the four variables (2D conductivity model, electrojet position, $1 \mathrm{D}$ conductivity model and electrojet strength) in our simulations which most affects the size of GIC. Hence, we examine the relative effect of each variable on the GIC using analysis of variance (ANOVA) for all 695 nodes. As many of the nodes have relatively small GIC overall $(<5$
A), we also examine the 12 nodes with the largest average GIC. We are interested in finding general conclusions as well as checking for local or individual effects, so we plot the GIC values for each node in geographical form to look for regional variation.

ANOVA is a statistical technique which can be used to discern the relative influence of different variables on the outcome of an experiment (e.g. Snedecor and Cochran 1989). In this case, there are four varying parameters, so the anovan function in Matlab is used to separate the 
influence of each by comparing their relative $F$ statistic to determine which is the most important. For each node, the 504 GIC values, along with three out of the four parameters relating to each GIC value are passed to the anovan function to compute the $F$ value, which is the main output statistic of interest. The parameter with the largest significant $F$ value was considered to be the most influential on the size of GIC generated at any particular node. This was then repeated for all four combinations of the variables:

1. 2D conductivity model, electrojet position, 1D conductivity model;

2. 2D conductivity model, 1D conductivity model, magnetic field strength;

3. 2D conductivity model, electrojet position, magnetic field strength;

4. 1D conductivity model, electrojet position, magnetic field strength.

From the $F$ value at each node, the most important parameter was chosen as the one occurring most frequently in the above four combinations. For example, if electrojet position was the important variable from combinations 1, 3 and 4, while strength was the most important in combination 2 , then the electrojet position has a dominant influence on the size of GIC generated at that particular node. Hence, it is recorded as the dominant parameter.

Table 3 gives a summary of the dominant parameters for each node as computed from the ANOVA. The first row gives the influence of each of the parameter using all 695 nodes from all 504 electric field models. Note, there are 25 nodes which exhibit practically zero GIC in all of the simulations. Five of these are 'virtual', in the sense that they represent line splits in the network, while the remainder are located too closely together to allow large GIC to flow. The majority of nodes (432) are influenced by the position of the electrojet in the simulations. For 69 nodes, it is the influence of the 2D conductivity model that is prevalent, while the $1 \mathrm{D}$ conductivity model has the least influence in overall statistics (32 nodes). Figure 5 shows the geographical distribution of the nodes associated with each parameter's influence.
The second row of Table 3 illustrates the dominant parameter if the problem is reduced to just the relative influence of the $2 \mathrm{D}$ versus $1 \mathrm{D}$ conductivity models by fixing the electrojet position (over the Irish Sea) and the magnetic field strength (at 200-year return at 10-min period). In this case, the GIC at more nodes are sensitive (488) to the 1D model than the 2D model (180). Figure 6 shows the geographical spread of the nodes. For the 2D models, the region around southern Scotland and northern England shows the greatest density of nodes, though lines of nodes lying along some of the conductivity features in the BGS2012 model are visible in the southern part of the UK.

The influence of the parameters on the 12 nodes with the largest GIC is examined in rows 3 and 4 of Table 3 . Including all the parameters (row 3 ) shows the electrojet position which controls the size of GIC, while fixing the electrojet position and strength, as before, indicates that the $1 \mathrm{D}$ conductivity models are dominant. Note that if we do vary the fixed electrojet position (i.e. for the statistics in row 2 ), then the $1 \mathrm{D}$ conductivity remains the dominant factor.

\section{Influence of conductivity models}

We next examine the variation from the 1D and 2D models in the top 12 nodes, to determine which conductivity model has the largest influence. To do this, we plot the GIC values out as a series of 'box' plots. Box plots show the range of the data in the form of a central box giving the 25 to 75 percentiles, with whiskers extending to the 9 to 91 percentiles. The line in the middle of the box shows the median value. Note that if the median between models is not different at the $5 \%$ significance level, their notched intervals will overlap. For clarity, the outlier values are not shown in these plots.

Figure 7 shows the box plots for the seven 1D conductivity models (c.f. Table 1 for acronyms). The letters A to $\mathrm{L}$ refer to the locations shown in Figure 2 and are ordered approximately by latitude from north to south. The box plots suggest that the largest GIC are generated by the homogeneous half-space and Northern Highland models, while the Southern Uplands model generates the smallest GIC values. This concurs with the electric field images

Table 3 Frequency of the most influential variable for GIC

\begin{tabular}{|c|c|c|c|c|c|}
\hline & $\mathrm{GIC} \leq 5 \mathrm{~A}$ & Cond 2D & Ejet Pos. & Cond 1D & Strength \\
\hline All nodes & 25 & 69 & 432 & 32 & 137 \\
\hline Fixed ejet/strength ${ }^{a}$ & 27 & 180 & - & 488 & - \\
\hline Top 12 nodes & - & 2 & 6 & 3 & 1 \\
\hline Fixed ejet/strength ${ }^{a}$ & - & 3 & - & 9 & - \\
\hline
\end{tabular}

${ }^{a}$ Irish Sea; 200-year, 10-min period, 21 electric field models only. 

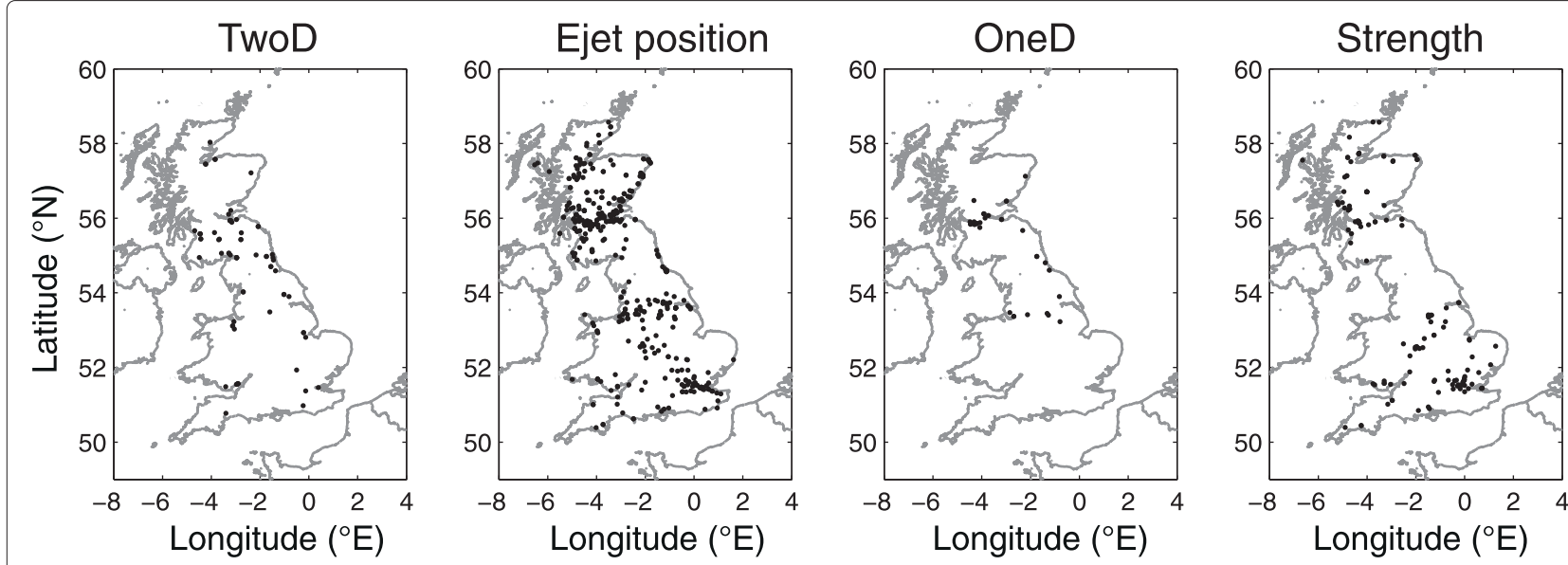

Figure 5 The most influential parameter at each of the 695 nodes.

shown in Figure 3. The 1D model has the largest influence on the generation of electric field values and hence GIC.

Figure 8 shows the spread of GIC data for the same nodes by splitting the dataset into values from the $2 \mathrm{D}$ conductivity models. The AMK2003 model shows lower GIC values in nodes A to $F$ (more northerly) while the BGS2012 model has lower GIC values for nodes G to L (more southerly). In certain nodes, the local conductivity can make a large difference. For example, in node C, the variation between GIC from the AMK2003 and the BGS2012 model is much larger. However, in general, the variation of GIC between 2D models is similar. The differences between the GIC in northern nodes (A to F) versus southern nodes ( $G$ to $L$ ) suggests that the BGS2012 2D model is correctly redistributing the electric field, as the more resistive lithology in the northern half of the region creates larger GIC compared to the more conductive geology of the southern regions (though with local exceptions).

Thus, at most nodes, the variation in GIC between the seven 1D models is larger than the variation between the three 2D models. Note that there are 168 values for each of the box plots in Figure 8, so the largest value outliers are not shown and the median and 9 to 91 percentile ranges will differ from Figure 7 which have only 72 values per box.

\section{Discussion}

The statistics from the analysis of variance method show that the electrojet position and strength are by far the
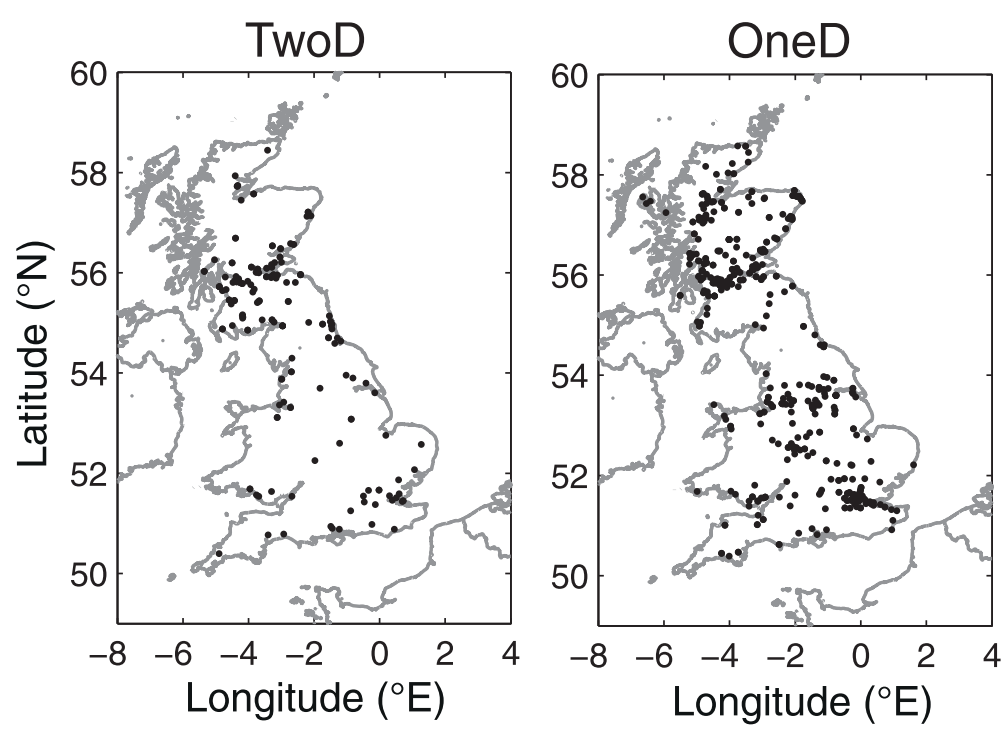

Figure 6 The more influential parameter of the two-dimensional (TwoD) or one-dimensional (OneD) conductivity model. 

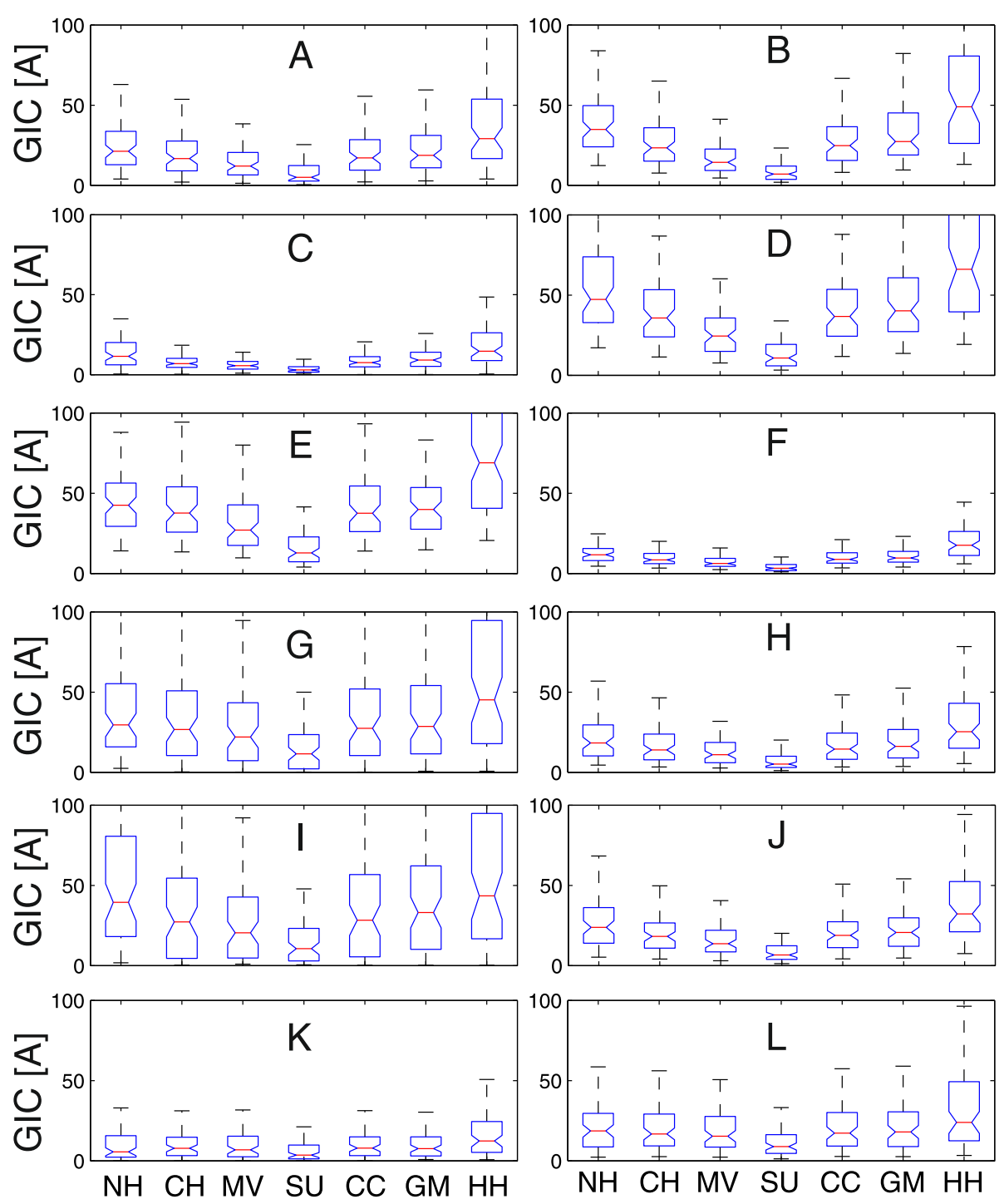

Figure 7 Box plots for the seven 1D conductivity models at the 12 nodes with largest GIC. Panels (A-L) refer to the nodes shown in Figure 2. See text for details and Table 1 for model acronyms.

dominant factors in the size of GIC values generated in the synthetic electric field models (c.f. Figure 5). Although this is not a new result, it does confirm previous findings and suggests that, in general, the conductivity structure is a second-order effect.

However, when examined in detail, it can be seen that in certain regions, variations in the 1D and 2D conductivity can enhance or diminish the GIC values particularly where the largest GIC are found. For example, the 2D AMK2003 model has a high conductivity structure in the central Scotland area, which gives lower GIC values than the other two models. In this area, the conductivity model is the dominant factor, leading to smaller GIC compared to the other models. The influence of the $2 \mathrm{D}$ conductivity models can be seen in Figure 6 where some of the nodes lie along more conductive lithologies in the southern part of the UK.

The 1D conductivity structure has a generally stronger influence and can lead to large differences in the GIC. In Figure 7, nodes D, E and G show much larger GIC when a homogeneous half-space value is used for the 1D conductivity, compared to the Southern Uplands model. In contrast, nodes $\mathrm{K}$ and $\mathrm{L}$ along the south coast are less sensitive to either 1D or 2D conductivity differences. Beggan et al. (2013) used the homogeneous half-space for their GIC analysis, and this has proven to be the most extreme form of $1 \mathrm{D}$ conductivity model.

Figure 3 shows that the most important surface variations occur on the boundaries of the conductivity contrasts, i.e. the coastline is obvious in many of the electric 


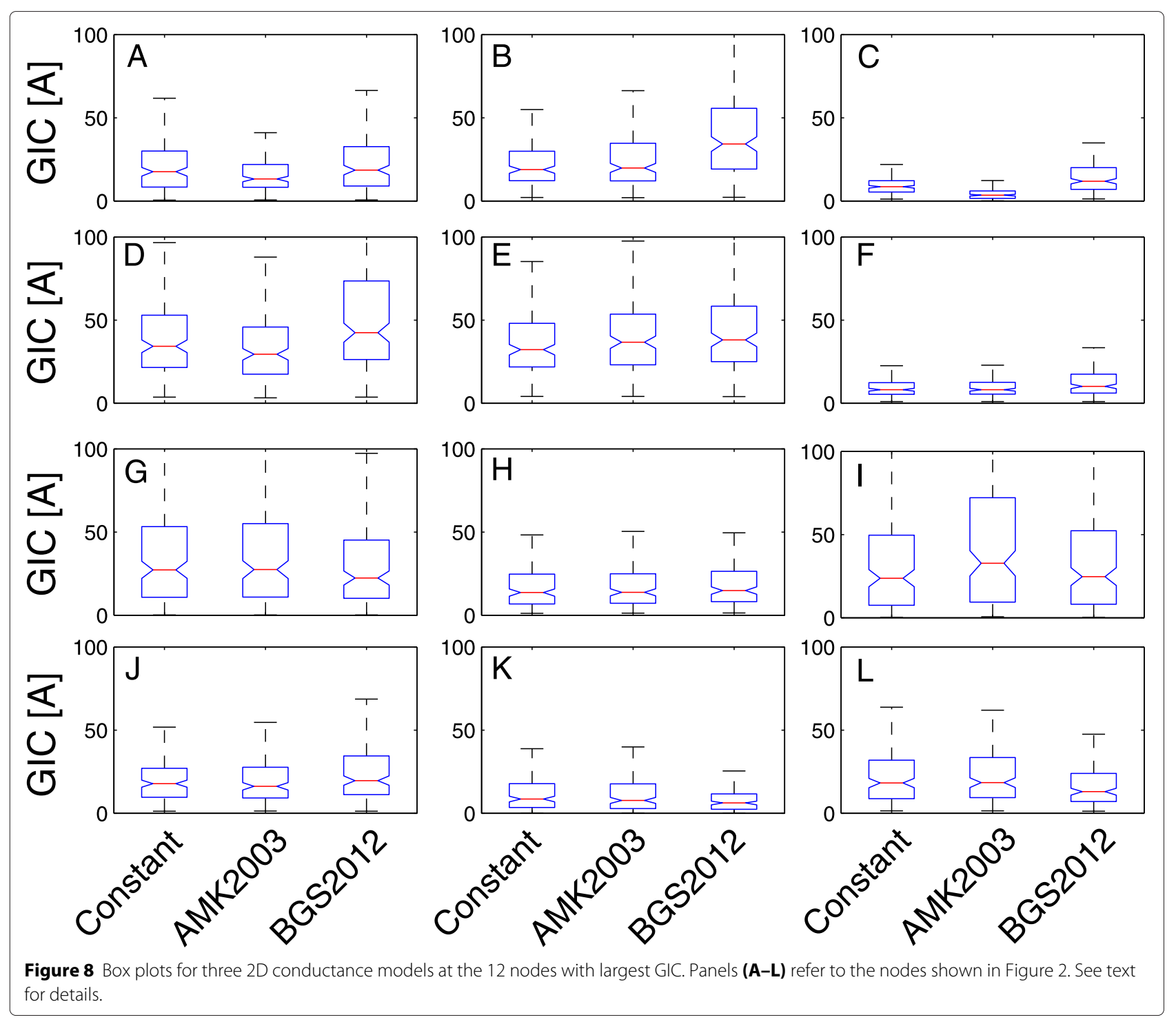

field models. As the UK is an island, this is the most important consideration for first-order modelling of GIC. The second-order differences are provided by the redistribution of the electric field by the variation within the 2D models, a feature noted in Beamish et al. (2002) for example.

Another point we wish to make is that even when the position of the electrojet is relatively distant, large GIC can occur. From Figure 4, it can be observed that when the electrojet is located over North Scotland, there are significant GIC along the coastal nodes in the east and west of the southern UK. This is likely to be a consequence of the topology of the UK network, whereby GIC outflows are dispersed from the source region along the least resistive parts of the high-voltage network.

We suggest further analysis should be made using measurements of GIC across the UK in key nodes when they become available. From such measurements, it is then possible to invert for conductivity (Vasseur and Weidelt 1977) and hence iteratively improve our knowledge of the conductivity structures affecting the nodes.

\section{Conclusions}

In this analysis, we are mainly concerned with identifying factors related to conductivity that may generate additional uncertainties in GIC which have not previously been accounted for. By examining three different 2D surface conductance models and seven different 1D conductivity depth models, we have attempted to assess some of the geologically feasible variations possible within the UK. We find that the major driver of GIC is the relative position and strength of the electrojet over the UK, while the influence from conductivity tends to be a second-order 
effect, in general, but can enhance or reduce local GIC in certain regions.

We have produced a series of 504 synthetic surface electric field scenarios for modelling GIC in the UK. The scenarios represent a best guess for various reasonable 'worst-case' scenarios during a large geomagnetic storm. The UK power grid is very well-connected and has a complicated topology giving it good resilience to the flow of large GIC. The multiple pathways through which currents can dissipate means that there are few nodes that are obviously vulnerable to major events, even given the uncertainties in our current modelling techniques.

\section{Competing interests}

The author declares that he has no competing interests.

\section{Acknowledgements}

The author thanks Alan Thomson, Gemma Kelly and Andrew Richards for discussions and suggestions on improving the manuscript and National Grid UK for network data and parameters. I also acknowledge the thorough comments and suggestions from the two reviewers, whose help was crucial. This research was partly funded by NERC New Investigators grant NE/J004693/1 and from the European Community's Seventh Framework Programme (FP7/2007-2013) under grant agreement 260330. This paper is published with the permission of the Executive Director of the British Geological Survey (NERC).

Received: 8 September 2014 Accepted: 6 December 2014 Published: 15 February 2015

\section{References}

Banks RJ, Beamish D, Geake MJ (1983) Magnetic variation anomalies in northern England and southern Scotland. Nature 303:516-518. doi:10.1038/303516a0

Banks RJ, Livelybrooks D, Jones P, Longstaff R (1996) Causes of high crustal conductivity beneath the lapetus suture zone in Great Britain. Geophys J Int 124:43-455. doi:10.1111/j.1365-246X.1996.tb07031.x

Beamish D (2012) The 1:625k near-surface bedrock electrical conductivity map of the UK. Technical Report OR/12/037, British Geological Survey, pp 23. http://nora.nerc.ac.uk/20833/

Beamish D, White J (2012) Mapping and predicting electrical conductivity variations across southern England using airborne electromagnetic data. Q J Eng Geol Hydrogeology 35:99-110. doi:10.1144/1470-9236/11-026

Beamish D, Clark TDG, Clarke E, Thomson AWP (2002) Geomagnetically induced currents in the UK: geomagnetic variations and surface electric fields. J Atmos Terr Phys 64:1779-1792

Beggan CD, Beamish D, Richards A, Kelly GS, Thomson AWP (2013) Prediction of extreme geomagnetically induced currents in the UK high-voltage network. Space Weather 11:407-419. doi:10.1002/swe.20065

Bernabeu EE (2013) Modeling geomagnetically induced currents in dominion Virginia power using extreme 100-year geoelectric field scenarios - part 1. IEEE Trans Power Delivery 28:516-523. doi:10.1109/TPWRD.2012.2224141

Boteler DH, Lackey AJC, Marti L, Shelemy S (2013) Power and Energy Society General Meeting (PES). In: Equivalent circuits for modelling geomagnetically induced currents from a neighbouring network. IEEE, New York. pp 1-5

Cannon P (2013) Extreme space weather: impacts on engineered systems and infrastructure. Technical report, Royal Academy of Engineering, London. ISBN 1-903496-95-0. www.raeng.org.uk/spaceweather

Lehtinen M, Pirjola R (1985) Currents produced in earthed conductor networks by geomagnetically-induced electric fields. Ann Geophysicae 4:479-484

McKay A (2003) Geoelectric fields and geomagnetically induced currents in the United Kingdom. PhD thesis, University of Edinburgh

Pirjola R (2005) Effects of space weather on high-latitude ground systems. Adv Space Res 36(12):2231-2240. doi:10.1016/j.asr.2003.04.074

Pulkkinen A, Bernabeu E, Eichner J, Beggan C, Thomson A (2012) Generation of 100-year geomagnetically induced current scenarios. Space Weather 10:04003. doi:10.1029/2011SW000750
Radasky WA (2011) Overview of the impact of intense geomagnetic storms on the US high voltage power grid. In: IEEE International Symposium on Electromagnetic Compatibility. IEEE Conference Publications, New York. pp 300-305. doi:10.1109/ISEMC.2011.6038326

Schulte in den Bäumen H, Moran D, Lenzen M, Cairns I, Steenge A (2014) How severe space weather can disrupt global supply chains. Nat Hazards Earth Syst Sci, 14:2749-2759. doi:10.5194/nhessd-2-4463-2014

Snedecor GW, Cochran WG (1989) Statistical methods. 8th edn. Iowa State University Press, lowa City

Thomson AWP (2014) Geomagnetic observatories: monitoring the Earth's magnetic and space weather environment. Weather 69(9):234-237. doi:10.1002/wea.2329

Thomson AWP, Dawson EB, Reay SJ (2011) Quantifying extreme behaviour in geomagnetic activity. Space Weather 9:10001. doi:10.1029/2011SW000696

Thomson AWP, McKay AJ, Clarke E, Reay SJ (2005) Surface electric fields and geomagnetically induced currents in the Scottish Power grid during the 30 October 2003 geomagnetic storm. Space Weather 3:11002. doi:10.1029/2005SW000156

Turnbull K (2010) Modelling GIC in the UK. Astronomy \& Geophysics 51(5):25-26. doi:10.1111/j.1468-4004.2010.51525.x

Turnbull K (2011) Modelling of geomagnetically induced currents in the United Kingdom. PhD thesis, University of Lancaster

Vasseur G, Weidelt P (1977) Bimodal electromagnetic induction in non-uniform thin sheets with an application to the northern Pyrenean induction anomaly. Geophys J R Astr Soc 51:669-690

Viljanen A (2011) European project to improve models of geomagnetically induced currents. Space Weather 9:07007. doi:10.1029/20115W000680

Viljanen A, Pirjola R (1994) Geomagnetically induced currents in the Finnish high-voltage power system. Surveys Geophys 15(4):383-408. doi:10.1007/BF00665999

Viljanen A, Pirjola R, Prácser E, Katkalov J, Wik M (2014) Geomagnetically induced currents in activity - modelled occurrence in a continent-wide power grid. J Space Weather Space Clim 4:09. doi:10.1051/swsc/2014006

\section{Submit your manuscript to a SpringerOpen ${ }^{\circ}$ journal and benefit from:}

- Convenient online submission

- Rigorous peer review

- Immediate publication on acceptance

- Open access: articles freely available online

- High visibility within the field

- Retaining the copyright to your article

Submit your next manuscript at $>$ springeropen.com 